\title{
Endocrine-Stromal Cell
}

National Cancer Institute

\section{Source}

National Cancer Institute. Endocrine-Stromal Cell. NCI Thesaurus. Code C41608.

A cell that forms the supporting matrix of one of the glands that secretes substances into the blood or lymph. 